\title{
C-reactive protein in bladder cancer: where do we stand?
}

Georgios Gakis and Arnulf Stenzl

In their Review, Saito and Kihara provided an in-depth discussion of the status of the prognostic role of serum C-reactive protein (CRP) in various urological cancers (C-reactive protein as a biomarker for urological cancers. Nat. Rev. Urol. 8, 659-666; 2011). ${ }^{1}$ We agree that, given CRP might indicate cancer-specific outcomes, its role in different cancer entities for which immunogenic factors might be involved is a relevant issue for prognosis. CRP is released by hepatocytes to activate the complement system in response to IL-6 secretion, which is produced by immune cells to facilitate cancer cell detection and opsonization. ${ }^{2}$ This process is integral to the immune-mediated response to the cancer microenvironment, ${ }^{3}$ which itself resembles a state of chronic inflammation with the aim of infiltrating the blood and lymphatic systems to form distant metastases. A variety of cytokines (such as IL-6) and growth factors are released to promote cytological crosstalk between the humoral and cytotoxic immune pathways to enhance the elimination of cancer cells.

Consequently, investigating the prognostic role of CRP in patients with cancer is a promising avenue for research. Essentially, serum CRP is a sensitive serological biomarker of acute or chronic inflammation that can be routinely assessed at low cost. Although CRP is mostly known in the medical community as a sensitive inflammatory marker indicating systemic response to pathogens, its prognostic potential as a tumour marker has been recently investigated in a subset of nonurological malignancies, including pancreatic, gastric, lung and ovarian cancer.

To clinically establish the prognostic use of serum CRP in urogenital cancers, however, one needs to investigate in depth its role in cancers with high immunogenicity. In fact, urothelial cancer is considered to be a highly immunogenic tumour, ${ }^{1}$ an assumption that is supported by the fact that bladder cancer cells penetrating the basement membrane and invading the subepithelial layer can be arrested (in terms of migration and cell division) by intravesical instillation with BCG. Instillation provokes local inflammation in the bladder wall and the resulting immune cells attack urothelial cells, resulting in increased cancer-cell elimination. ${ }^{4}$ Thus, the degree of systemic inflammation might reflect the likelihood the cancer will invade deeper layers of the bladder and form metastases.

We recently investigated the role of serum $\mathrm{CRP}$ in patients undergoing radical cystectomy for muscle-invasive bladder cancer. ${ }^{5}$ We found that preoperative serum CRP was an independent risk factor for cancerspecific survival. Elevated levels were strongly associated with locally advanced tumour stage ( $\geq$ pT3a), lymph node density and resection margin status. To determine its prognostic power, we incorporated CRP into a novel prognostic model-termed TNR-C (tumour stage-node densityresection margin-CRP) Score-and demonstrated a statistically significant enhancement of predictive accuracy (by $4.9 \%$ ) by adding CRP to a basic prognostic model encompassing major pathological parameters of survival.

Although serum CRP is a unique and sensitive biomarker of acute or chronic inflammatory response, it lacks specificity. This drawback is the reason why the implementation of serum CRP as a biomarker for cancer is difficult to achieve. Increased serum CRP levels can potentially be the result of any intervention prior to radical cystectomy as well as subclinical infection. To adjust for this bias, we investigated whether the time interval between the last transurethral resection and radical cystectomy, the number of previous transurethral resections or the preoperative presence of leukocytosis were associated with CRP levels. None of these three factors were significantly correlated with CRP, which suggests that these possible confounders do not have a role in the interpretation of CRP levels. ${ }^{5}$ These findings, therefore, further strengthen the role of CRP as an oncological biomarker in muscle-invasive bladder cancer.

Finally, we must consider what (if any) clinical applications can be developed for serum CRP in urothelial cancer. Although radical cystectomy is considered the treatment of choice for muscle-invasive bladder cancer, the inauguration of neoadjuvant chemotherapy has provided scope for further improving outcomes in these patients. However, despite level I evidence derived from prospective randomized trials in favour of such an approach, ${ }^{6}$ neoadjuvant chemotherapy has not been adopted into daily clinical routine-only $17 \%$ of patients treated (even in a tertiary referral centre) received perioperative chemotherapy before radical treatment. ${ }^{7}$ This reluctance of practitioners to endorse neoadjuvant therapy might be because of a lack of indicators (such as cross-sectional imaging techniques) that can accurately predict which patients will benefit most from its implementation. Furthermore, a compounding fear that any delay in radical cystectomy in those who do not respond to chemotherapy might further worsen outcomes might also prevent widespread use of this perioperative therapy. ${ }^{8}$ Assessing serum CRP levels might offer an advantage over tissue-based markers, which can be altered during treatment, and might be associated with tumour response. Thus, CRP is a potential rational real-time marker for use in the neoadjuvant and adjuvant settings. ${ }^{9}$

Department of Urology, University Hospital Tübingen, Eberhard-Karls University, HoppeSeyler Strasse 3, D-72076 Tübingen, Germany (G. Gakis, A. Stenzl).

Correspondence to: G. Gakis georgios.gakis@web.de

doi:10.1038/nrurol.2011.145-c1 
Competing interests

The authors declare no competing interests.

1. Saito, K. \& Kihara, K. C-reactive protein as a biomarker for urological cancers. Nat. Rev. Urol. 8, 659-666 (2011).

2. Mantovani, A., Allavena, P., Sica, A. \& Balkwill, F. Cancer-related inflammation. Nature 454, 436-444 (2008).

3. Balkwill, F. \& Mantovani, A. Inflammation and cancer: back to Virchow? Lancet 357, 539-545 (2001).

4. Ardelt, P. U. et al. Reactive antibodies against Bacillus Calmette-Guerin heat-shock protein-65 potentially predict the outcome of immunotherapy for high-grade transitional cell carcinoma of the bladder. Cancer 116, 600-609 (2010).

5. Gakis, G. et al. Development of a new outcome prediction model in carcinoma invading the bladder based on preoperative serum C-reactive protein and standard pathological risk factors: the TNR-C score. BJU Int. 108, 1800-1805 (2011).

6. Grossman, H. B. et al. Neoadjuvant chemotherapy plus cystectomy compared with cystectomy alone for locally advanced bladder cancer. N. Engl. J. Med. 349, 859-866 (2003).
7. Raj, G. V. et al. Contemporary use of perioperative cisplatin-based chemotherapy in patients with muscle-invasive bladder cancer. Cancer 117, 276-282 (2011).

8. Alva, A. S. et al. Efficient delivery of radical cystectomy after neoadjuvant chemotherapy for muscle-invasive bladder cancer: a

multidisciplinary approach. Cancer 118, 44-53 (2012).

9. Gakis, G., Schwentner, C., Todenhoefer, T. \& Stenzl, A. Current status of molecular markers for prognostication and outcome in invasive bladder cancer. BJU Int. 110, 233-237 (2012). 